\title{
Estudio sobre la Velocidad y Tiempo de Biodegradación Bajo Condiciones Controladas de Fibras Naturales de Fique (Furcraea andina) y Algodón (Gossypium barbadense)
}

\author{
Yamid A. Pinchao-Pinchao ${ }^{1}$, Oswaldo Osorio-Mora ${ }^{1 \star}$, Oscar Checa-Coral ${ }^{2}$, y Elizabeth Tobar ${ }^{1}$ \\ (1) Facultad de Ingeniería Agroindustrial, Departamento procesos industriales, Universidad de Nariño, Grupo de \\ Investigación GAIDA, San Juan de Pasto-Colombia (Correo-e: yamidalexis@hotmail.com; \\ osorio_oswaldo@hotmail.com; elizabetth.t93@gmail.com); \\ (2) Facultad de ingeniería agronómica, Universidad de Nariño, Grupo de investigación en cultivos andinos GRICAND, \\ San Juan de Pasto-Colombia (Correo-e: gricandudenar@gmail.com)
}

Recibido Ago. 2, 2018; Aceptado Oct. 16, 2018; Versión final Ene. 17, 2019, Publicado Ago. 2019

\begin{abstract}
Resumen
Se determinó el tiempo y porcentaje de biodegradación de fibras de fique, algodón y polipropileno mediante la metodología de la Norma ASTM 5988-12. La degradación del polipropileno empleado en cultivo de arveja en Nariño genera problemas ambientales, razón que motiva este estudio. Paralelamente, se hizo análisis de textura de las fibras expuestas a condiciones ambiente reales. $\mathrm{El} \mathrm{CO}_{2}$ producido se atrapo con $\mathrm{KOH}$ y se calculó indirectamente por titulación con $\mathrm{HCl}$. Se asumió un orden de reacción cero para biodegradación de las fibras. Las velocidades y tiempo de biodegradación fueron $0.547,0.111,0.0147 \%$ /día y 6.06, 29.90, 225.20 meses para fique, algodón y polipropileno respectivamente. La tensión a carga máxima mostró que la fibra de fique bajó de 100 a $26 \mathrm{MPa}$ en 16 días, mientras que el algodón y polipropileno no perdieron esta propiedad. Finalmente, las fibras de fique y algodón serían las más adecuadas para emplear en tutorado de arveja.
\end{abstract}

\section{Study on the Rate and Time of Biodegradation under Controlled Conditions of Natural Fibers of Fique (Furcraea andina) and Cotton (Gossypium barbadense)}

\begin{abstract}
Time and percentage of biodegradation of fique, cotton and polypropylene fibers was determined by the methodology of ASTM 5988-12. The degradation of the polypropylene used in pea crop in Nariño generates environmental problems, reason that motivates this study. In parallel, texture analysis of the fibers exposed to real environmental conditions was done. The $\mathrm{CO}_{2}$ produced was trapped with $\mathrm{KOH}$ and indirectly calculated by titration with $\mathrm{HCl}$. A zero-reaction order was assumed for biodegradation of the fibers. The velocities and biodegradation time were $0.547,0.111,0.0147 \%$ /day and $6.06,29.90,225.20$ months for fique, cotton and polypropylene respectively. The tension at maximum load showed that the fique fiber decreased from 100 to $26 \mathrm{MPa}$ in 16 days, while cotton and polypropylene did not lose this property. Finally, the fibers of fique and cotton would be the most suitable to use in pea crop.
\end{abstract}




\section{INTRODUCCIÓN}

En el departamento de Nariño el cultivo de arveja es una de las principales actividades agrícolas con un área de siembra de 17.000 hectáreas. Sin embargo, el manejo de este cultivo requiere sistemas de tutorado robustos que emplean 146 conos de fibra de polipropileno por hectárea de arveja tutorada. Un cono tiene aproximadamente $5 \mathrm{~km}$ de fibra, lo que significa que por cada hectárea de producción de arveja se desechan $730 \mathrm{~km}$ de fibra de polipropileno, representando una problemática ambiental generalizada. Además, de la contaminación del suelo, la alta durabilidad del polipropileno representa un factor de riesgo principalmente para el ganado bovino y ovino (Gómez y Michel, 2013). Esto, debido a que, en los campos Nariñenses aun es común que los agricultores realicen actividades de pastoreo en los lotes empleados para cultivo de arveja. Ante esta situación, se hace necesario la búsqueda de otro tipo de materiales para el tutorado de arveja como las fibras naturales, no sin antes evaluar sus tiempos de biodegradabilidad como requisito previo y necesario para extender su aplicación en los cultivos de arveja (Lucas et al., 2008).

En la actualidad el uso de materiales poliméricos de propiedades mecánicas y características tales como bajo costo, durabilidad y procesabilidad, ha generado una gran cantidad de aplicaciones de estos, provocando un aumento en los flujos de desechos y mala disposición (Emadian et al., 2017). En los cultivos de arveja la situación no es indiferente, de hecho, se ha determinado que el polipropileno en un lapso de 2 años bajo biodegradación controlada apenas alcanza el $1 \%$ de biodegradación, lo que, genera graves consecuencias ambientales ( Gómez y Michel, 2013). En consecuencia, se ha prestado mucha atención a los materiales de origen natural como alternativas sostenibles de recursos renovables que mitiguen los impactos ambientales (Emadian et al., 2017). Ante esta situación las fibras naturales llegarían a ser un posible sustituto al hilo de polipropileno que se emplea una sola vez por cultivo de arveja durante el tutorado y se deshecha (Arpitha y Yogesha, 2017).

Por su parte, las fibras de fique y algodón provienen de fuentes naturales renovables que las convierte en una alternativa amigable con el ambiente para la sustitución de fibras derivadas del petróleo en el tutorado de arveja, ya que, poseen propiedades mecánicas similares (Kicińska-Jakubowska et al. 2012; Milošević et al. 2017). No obstante, para determinar su posible uso en sistemas de tutorado es necesario realizar pruebas de textura y biodegradación, con el fin de determinar su impacto ambiental después de uso. En este sentido se puede emplear diferentes técnicas cuantitativas y cualitativas para evaluar la biodegradación como: observaciones visuales, mediciones de pérdida de peso, cambios en las propiedades mecánicas, evolución de $\mathrm{CO}_{2}$, entre otros (Milošević et al. 2017; Nair et al. 2017). Estos análisis, permitirán estimar si las fibras de algodón y fique representan alternativas de sustitución al polipropileno (Eubeler et al., 2009; Nair et al., 2017). Con respecto a la biodegradación puede ocurrir en condiciones aeróbicas en presencia de oxígeno y anaeróbicas sin oxígeno disponible, reduciendo los materiales a dióxido de carbono, agua, sales minerales y nuevos constituyentes celulares microbianos o biomasa (Gómez y Michel, 2013; ASTM D5988-12). Generalmente, la biodegradación se mide a través, de cinéticas de consumo de oxígeno o evolución del dióxido de carbono $\left(\mathrm{CO}_{2}\right)$ (Scott, 2015; ASTM D5988-12). El $\mathrm{CO}_{2}$ producido se puede medir de diferentes formas, una de ellas es la medición acumulativa en donde el $\mathrm{CO}_{2}$ producido queda atrapado en una solución, generalmente hidróxido de Bario $\left(\mathrm{Ba}(\mathrm{OH})_{2}\right)$ o hidróxido de potasio $(\mathrm{KOH})$ durante la prueba y luego se cuantifica por medio de titulación acido-base (ASTM D5988-12; Milošević et al. 2017). Bajo condiciones controladas en el laboratorio se ha realizado esta técnica revelando que la degradación a dióxido de carbono respecto al tiempo probablemente genere una curva con tres etapas, fase de retardo, fase de crecimiento y fase estacionaria (Komilis, 2006).

La velocidad y tiempo de degradación también están relacionadas directamente con la composición del material de prueba, es así como las fibras naturales son objeto de estudio para refuerzo de matrices poliméricas debido a ventajas sobre las fibras sintéticas tales como la renovabilidad, baja densidad y alta resistencia específica (Arpitha y Yogesha, 2017). Además, su composición biológica que generalmente es lignocelulósica produce gases, compuestos volátiles de baja densidad, cambios de olor y color, generación de dióxido de carbono y agua cuando ocurren procesos de degradación por rupturas de enlaces entre celulosa y lignina (Kicińska-Jakubowska et al., 2012). En este contexto, la determinación de la biodegradabilidad de un material es el paso clave para posteriores aplicaciones amigables con el ambiente; por lo tanto, el objetivo de esta investigación fue evaluar el porcentaje y tiempo de degradación de fibras naturales de fique y algodón bajo condiciones de laboratorio utilizando métodos de ensayo estandarizados para biodegradabilidad.

\section{MATERIALES Y MÉTODOS}

La investigación y análisis se realizaron en los laboratorios de Investigación en Conservación y Calidad de Alimentos y de estudios de biodegración de la Universidad de Nariño sede Torobajo, Pasto, Nariño, localizados a $2527 \mathrm{msnm}$., con una temperatura promedio de $14{ }^{\circ} \mathrm{C}$ y una humedad relativa de $70 \%$. 


\section{Obtención de la muestra y reactivos}

Las fibras de fique, algodón y polipropileno fueron obtenidas en el mercado Potrerillo de la ciudad de Pasto. El suelo empleado en los montajes de biodegradación fue recolectado en el centro de producción limpia LOPE SENA $(\mathrm{pH}$ inicial $=6.73, \mathrm{pH}$ final $=6.95$, relación C:N 14:1, capacidad de retención de humedad $=61 \%$, humedad $=48 \%)$. El ácido clorhídrico fumante $(\mathrm{HCl})$ y el hidróxido de potasio $(\mathrm{KOH})$ al $85 \%$ fueron obtenidos de Panreac.

\section{Montaje para el estudio de biodegradación}

El estudio de biodegradación se realizó acorde a la norma ASTM D5988-12. Se utilizó como sustrato $450 \mathrm{~g}$ de suelo con tamaño de partícula de $2 \mathrm{~mm}$ y humedad ajustada al $70 \%$ mas $50 \mathrm{~g}$ de compost estandarizado. Se pesó $1 \mathrm{~g}$ de cada fibra. El $\mathrm{CO}_{2}$ producido fue atrapado con $20 \mathrm{~mL}$ de $\mathrm{KOH} 0,5 \mathrm{~N}$ y para mantener la humedad de cada montaje se empleó $50 \mathrm{~mL}$ de agua destilada depositados en beakers de $100 \mathrm{~mL}$. Todos los materiales se ubicaron al interior de recipientes de vidrio de $4 \mathrm{~L}$ y se sellaron herméticamente. A su vez los montajes se depositaron en cajas de icopor, equipadas con bombillos que permiten mantener la temperatura al interior a $24 \pm 3{ }^{\circ} \mathrm{C}$. El montaje puede verse en la Fig. 1. El $\mathrm{CO}_{2}$ producido se estimó por titulación cada 4 días del $\mathrm{KOH}$ con $\mathrm{HCL} 0,25 \mathrm{~N}$ hasta alcanzar un pH 8,3 medido con el pH-metro Thermo Scientific ${ }^{\mathrm{TM}}$ Orion Star ${ }^{\mathrm{TM}}$ A111 Benchtop Meter. Se utilizó un control o blanco replicando el montaje en las mismas condiciones, pero sin ningún tipo de fibra. Los experimentos se condujeron por triplicado.
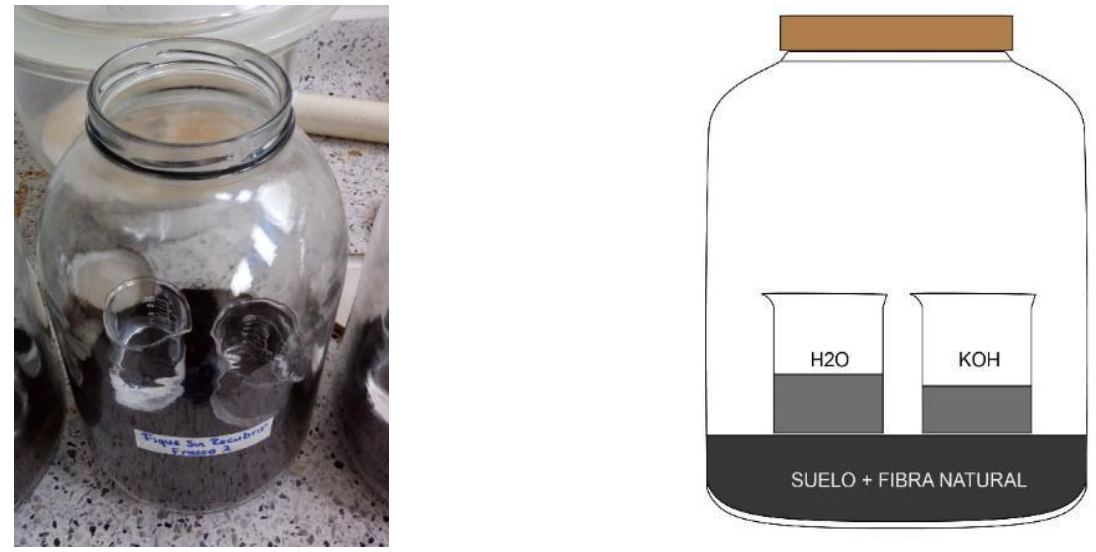

Fig. 1: Montaje para evaluación de la biodegradación de fibras naturales

Calculo del porcentaje de biodegradación de las fibras

Los resultados se expresaron cómo \% Biodegradación calculado como la relación entre los $\mathrm{g} \mathrm{CO}_{2}$ producido y g CO 2 teórico (ASTM D5988 - 12 2014; Leejarkpai et al., 2011). La cantidad de $\mathrm{CO}_{2}$ teórico fue calculado acorde a la Ec. 1. El porcentaje de biodegradación fue calculado conforme a la Ec. 2.

$$
\mathrm{CO}_{2} \text { teórico }=M_{T O T} \times C_{T O T} \times \frac{44}{12}
$$

Donde: $\mathrm{CO}_{2}$ teórico es la cantidad teórica de $\mathrm{CO}_{2}$ que puede producirse por las muestras de fibras en gramos en cada montaje; Мтот es el total de sólidos secos en gramos de las muestras de cada fibra añadida al inicio de la prueba a cada montaje; Стот es la proporción de carbono orgánico total en gramos presentes en el total de sólidos secos por cada gramo de fibra.

$$
\% \text { Biodegradación }=\frac{\left(\mathrm{CO}_{2}\right)_{M}-\left(\mathrm{CO}_{2}\right)_{B}}{\mathrm{CO}_{2} \text { teórico }} \times 100
$$

Donde: $\left(\mathrm{CO}_{2}\right)_{\mathrm{M}}$ es la cantidad acumulada de dióxido de carbono en gramos que se produjo en cada montaje que contienen las muestras de fibras; $\left(\mathrm{CO}_{2}\right)_{\mathrm{B}}$ es la cantidad de dióxido de carbono en gramos producida en el montaje del blanco. El $\mathrm{CO}_{2}$ de la muestra y el blanco se calcularon con la Ec. 3

$$
\mathrm{g} \mathrm{CO}_{2}=\frac{(0,5 \mathrm{~N}) \times \mathrm{mL} \mathrm{HCl} \times 44}{1000}
$$

Donde: $(0,5 \mathrm{~N})$ es la normalidad del $\mathrm{KOH} ; \mathrm{mL} \mathrm{HCl}$ son los mililitros gastados de ácido clorhídrico en la titulación del $\mathrm{KOH}$. 


\section{Estimación de la velocidad $k$ y modelamiento cinético de la de biodegradación de las fibras}

La velocidad y el modelamiento cinético se realizó acorde a Heldman (2011) quien sostiene que en la mayoría de las aplicaciones, la velocidad de reacción se establece midiendo la velocidad de desaparición de uno o más reactivos, o cuantificando la aparición de un producto de reacción. Adicionalmente, algunas reacciones de deterioro se describen mejor por la relación lineal entre la concentración de un producto y el tiempo. Por lo tanto, los cambios en la aparición del $\mathrm{CO}_{2}$ durante la biodegradación de las fibras naturales se modeló por la relación lineal entre el porcentaje de $\mathrm{CO}_{2}$ y el tiempo, es decir, mediante un modelo de orden cero. La expresión para la reacción de orden cero se describe en la Ec. 4 e integrando la Ec. 3 (Heldman, 2011; Labuza, 1984) se obtiene la Ec. 5 para reacciones de orden cero.

$$
\begin{aligned}
& \frac{+d[B]}{d t}=k_{0} \\
& {[B]-\left[B_{0}\right]=k_{0} t}
\end{aligned}
$$

Donde: el porcentaje de biodegradación (B) en cualquier momento (t) es una función del porcentaje de biodegradación inicial $\left(B_{0}\right)$ y la constante de velocidad de orden cero $\left(\mathrm{k}_{0}\right)$.

A partir de la Ec. 5 se determinó el tiempo que tomarían en degradarse las fibras hasta un $100 \%$ (Labuza, 1984). La constante de velocidad k se estimó de la gráfica de los datos experimentales de Biodegradación (\%) versus tiempo, donde a través de regresión lineal se tomó al valor de la pendiente como la velocidad $\mathrm{k}$ (Heldman, 2011).

\section{Observación superficial de las fibras naturales biodegradadas}

Paralelamente se realizó el seguimiento de la biodegradación bajo condiciones de ambiente normal de cultivo de arveja, es decir, a campo abierto con el fin de observar los cambios en la estructura superficial de cada fibra. Las muestras se enterraron en un lote ubicado en el centro de producción limpia LOPE SENA a una profundidad de $20 \mathrm{~cm}$, separadas a una distancia de $1 \mathrm{~m}$. Cada una de las muestras se extrajeron y observaron cada 8 días en un estereoscopio óptico ZEISS Axio Lab.A1 de imagen de Luz polarizada, con captura de imagen digital mediante la cámara AxioCam ICc1 y análisis de imagen mediante software ZEN. Adicionalmente, se midió la propiedad de tensión a carga máxima acorde con la norma ASTM D3822 en el Texturómetro LS1 (Test and Calibration Instruments Ametek, Lloyd Materials testing, USA) con distancia de separación de las tenazas de $100 \mathrm{~mm}$, distancia de recorrido de $100 \mathrm{~mm}$ y retorno automático.

\section{Análisis estadístico}

Se realizó análisis de varianza de las regresiones lineales simples con ayuda del software Stathgraphics Centurion XVI con un nivel de confianza del $95 \%$ y como parámetros de confiabilidad se tuvo en cuenta el coeficiente de correlación, el R-cuadrado y el R-cuadrado ajustado.

\section{RESULTADOS Y DISCUSION}

Los resultados obtenidos en los experimentos de biodegradación en laboratorio y a campo abierto se presentan a continuación.

\section{Cinéticas y estimación del tiempo de biodegradación de las fibras naturales}

En las Fig. 2, 3, y 4 se muestran los porcentajes de biodegradación alcanzados por las fibras de fique y algodón respectivamente durante un periodo de análisis de 116 días. Los puntos de color azul en cada figura muestran los datos promedio recolectados en cada ensayo y las líneas de colores indican la estimación realizada mediante regresión lineal con sus respectivos límites de predicción. En la Tabla 1, se muestra los parámetros cinéticos y el tiempo estimado para degradar las fibras en un $100 \%$. Los resultados muestran que la fibra de fique presenta un mayor porcentaje de biodegradación frente a la fibra de algodón y la fibra de polipropileno durante los 116 días de experimentación. Esto se debe especialmente a que la fibra de fique está compuesta por celulosa y lignina (UNCTAD, 2014). Estos elementos se superponen para formar filamentos multicelulares que dan estructura a la fibra de fique. La lignina disminuye la suavidad y flexibilidad haciendo que la textura del fique sea quebradiza, lo que facilita el ataque de microorganismos, que en condiciones de degradación aeróbica, oxidan la celulosa a dióxido de carbono que retorna a la atmósfera (Eriksson et al., 1990; Kicińska-Jakubowska et al., 2012). 


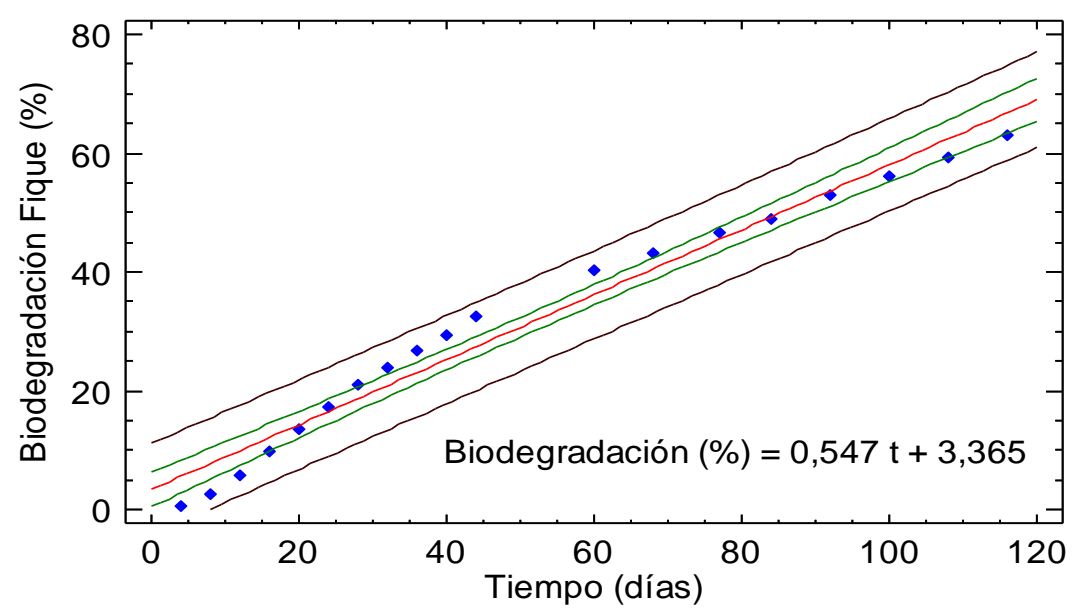

Fig. 2: \% de biodegración en fique versus tiempo, Coeficiente de Correlación = 0,99, R-cuadrada $=97,23 \%$, R-cuadrado ajustado $=97,07 \%$.

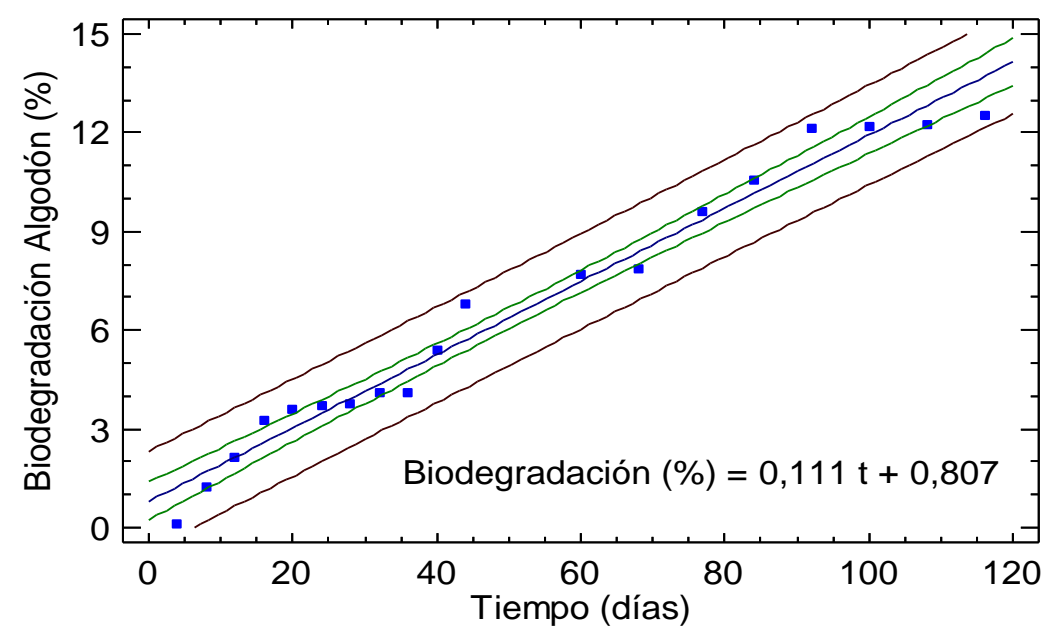

Fig. 3: \% de biodegración en algodón versus tiempo, Coeficiente de Correlación $=0,99$, R-cuadrada $=97,44 \%$, R-cuadrado ajustado $=97,29 \%$

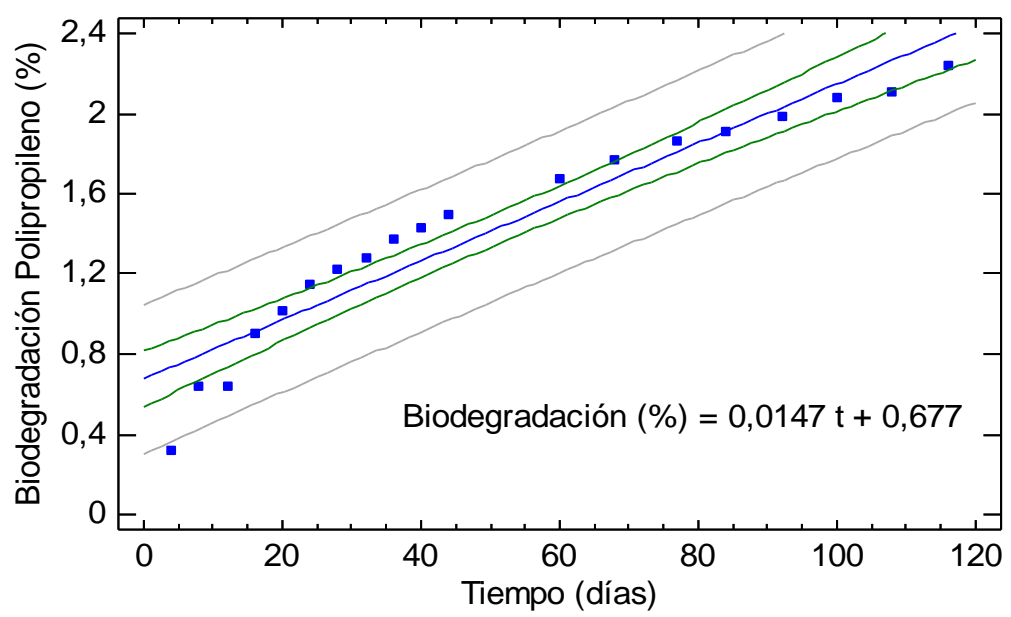

Fig. 4: \% de biodegración en polipropileno versus tiempo, Coeficiente de Correlación $=0,95$, R-cuadrada $=91,76 \%$, R-cuadrado ajustado $=91,28 \%$

En contraste, la fibra de algodón está compuesta solamente de celulosa, es una fibra altamente flexible y resistente en comparación con otras fibras de origen natural (Kicińska-Jakubowska et al., 2012; Blas-sevillano et al. 2018). En condiciones normales de temperatura es difícil de oxidar, autores como Milošević et al. (2017) han analizado esta fibra y determinaron que para hidrolizar esta celulosa constituyente del algodón hay que aplicar cizallamiento, adicionar enzimas y ácidos fuertes, de ahí que durante el proceso de biodegradación presente un menor porcentaje bajo las condiciones empleadas. 
Tabla 1: Ecuaciones, constante de velocidad y tiempo de biodegración de fique y algodón.

\begin{tabular}{|l|l|l|l|l|}
\hline Fibra & Ecuación lineal & Constante $k$ & Tiempo (días) & Tiempo (meses) \\
\hline Fique & Biodegradación $(\%)=0.547 \mathrm{t}+3.365$ & 0.547 & 182 & 6.1 \\
\hline Algodón & Biodegradación $(\%)=0.111 \mathrm{t}+0.807$ & 0.111 & 897 & 29.9 \\
\hline Polipropileno & Biodegradación $(\%)=0.0147 \mathrm{t}+0.677$ & 0.0147 & 6757 & 225.2 \\
\hline
\end{tabular}

En términos de cinéticas de degradación de estas fibras se puede observar en la Tabla 1, que la fibra de fique presento una mayor velocidad de biodegradación. Esto se debe a que diversos parámetros como la humedad, temperatura, $\mathrm{pH}$, salinidad, presencia o ausencia de oxígeno y nutrientes tienen una alta influencia sobre la degradación microbiana del fique (Arpitha y Yogesha, 2017). Además, dadas las características químicas y físicas de cada fibra como difusividad, porosidad, morfología, tipo de enlaces en las cadenas, pureza, reactividad química, fuerza mecánica, tolerancia térmica hacen que la fibra fique sea más susceptible al ataque de microorganismos que las fibras de algodón y polipropileno (Hughes, 2012; Nair et al., 2017).

A partir de los parámetros mostrados en la Tabla 1 y aplicando la ecuación de orden cero se estimó que el tiempo que tomaría en degradarse las fibras de fique, algodón y polipropileno en un $100 \%$ es de 6,06, 29,9, y 225 meses respectivamente bajo condiciones controladas de biodegradación. El corto tiempo que le toma a la fibra de fique y algodón se traduce en un impacto positivo para el medio ambiente en contraste con el polipropileno empleado en el cultivo arveja que tarda mucho tiempo en degradarse (Gómez y Michel, 2013). Luego, el polipropileno usado en los ensayos bajo condiciones experimentales controladas le tomaría hasta 18 años (225 meses) en degradarse. Esto se debe posiblemente a que, dadas sus propiedades químicas no se degrada por el ataque de microorganismos, así como tampoco se vería afectado por las condiciones ambientales (Jeon y Kim, 2016). Estudios como el de Jeon y Kim (2016) demuestran que incluso el polipropileno reducido a partículas de polvo y sometido a degradación por $S$. panacihumi PA3-2 alcanza un $20.3 \pm 1.39 \%$ durante 90 días. Así mismo, Gómez y Michel (2013) mostraron que el polipropileno en un lapso de 2 años bajo biodegradación controlada apenas alcanza el $1 \%$. Por esta razón, la fibra de fique y algodón representan una gran alternativa al uso de polímeros derivados del petróleo para uso en el tutorado de arveja debido a su rápida biodegradabilidad. Cabe resaltar que las cinéticas de biodegración de las fibras fueron realizadas en un rango de tiempo entre los 4 y 116 días, es decir, que hasta los 116 días las cinéticas de biodegradación se comportan de forma lineal. No obstante, es probable que después de este tiempo la biodegradación de las fibras de algodón y fique alcancen las mesetas de biodegradación. Adicionalmente, como sostiene Chattopadhyay et al. (2011) es posible que las fibras no lleguen a degradarse en un $100 \%$, no obstante, para fines pertinentes de expresión de tiempo los cálculos de tiempo se basan en un teórico ideal de $100 \%$ de biodegradación.

\section{Observación óptica de las fibras}

En las Fig. 5 se muestran los cambios externos estructurales presentados por las fibras de fique, algodón y polipropileno durante los 96 días de experimentación a campo abierto. Se puede observar que hasta los 64 días se registró presencia de fibra de fique y 8 días después (72 días) ya no se encontró residuos de este producto, esto se debe a que, la fibra de fique se biofracmentó por completo entre los 64 y 72 días. Estos datos visuales del fique comparados con los análisis de biodegradación a nivel de laboratorio muestran que la producción de $\mathrm{CO}_{2}$ en el montaje de fibra de fique hasta el día 116 apenas ha alcanzado un $60 \%$ de biodegradación. Lo anterior puede explicarse por la conformación de la estructura de la fibra de fique, es decir, estructuralmente ya no se visualiza a la fibra, debido a que este polímero ha sido degradado en oligómeros, dímeros y monómeros de bajo peso molecular, los cuales finalmente se mineralizan en $\mathrm{CO}_{2}$ y $\mathrm{H}_{2} \mathrm{O}$ ( $\mathrm{Nair}$ et al., 2017; Oliveira et al., 2015). De hecho, estudios como el de Gómez y Michel (2013) muestran que después de 200 días de análisis de biodegradación de fibras naturales reforzadas con polímeros continúan generando $\mathrm{CO}_{2}$ provocado por el proceso de mineralización final de las fibras.

Por su parte, las fibras de algodón y de polipropileno mantienen su estructura principal sin cambios observables en los 96 días de experimentación. En el caso del algodón, se debe a que, está compuesto en un $96 \%$ por celulosa y esta a su vez presenta un mayor grado de polimerización y cristalinidad ( $D P=3133$ y $\mathrm{Cl}=92 \%$ respectivamente) que la celulosa presente en el fique (Blas-sevillano et al. 2018; De Silva y Byrne 2017). Por tal razón, la resistencia y durabilidad del algodón aumenta. Además, el sistema de hilado y los enlaces de hidrogeno de los grupos hidroxilo de áreas cristalinas de las cadenas de celulosa dentro de las fibras de algodón le permiten al hilo mantenerse compacto en su estructura (Blas-sevillano et al. 2018; De Silva y Byrne 2017). Esto puede verse claramente en la Fig. 5 donde el hilo de algodón en la observación a los 96 días mantiene su configuración estructural. De igual forma, la fibra de polipropileno es altamente resistente debido a que posee una estructura semicristalina, con un grado de cristalinidad de $41 \%$ (Jmal et al. 2018). En tal sentido, las regiones cristalinas le otorgan resistencia, y las regiones amorfas le dan flexibilidad y consistencia (Coreño-Alonso y Méndez-Bautista 2010). Es por esto, que la fibra de fique en los 96 días de observación mantiene su estructura intacta en comparación con las fibras de fique y algodón. 
días

8
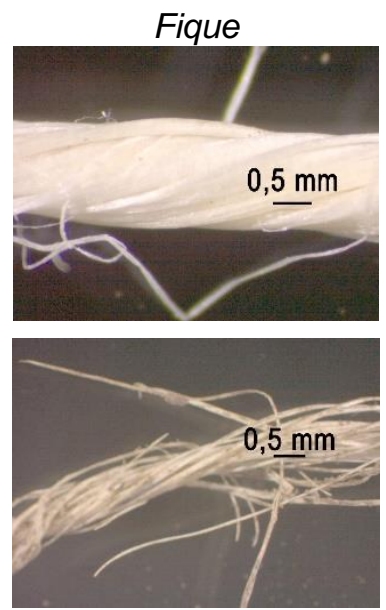

40


96
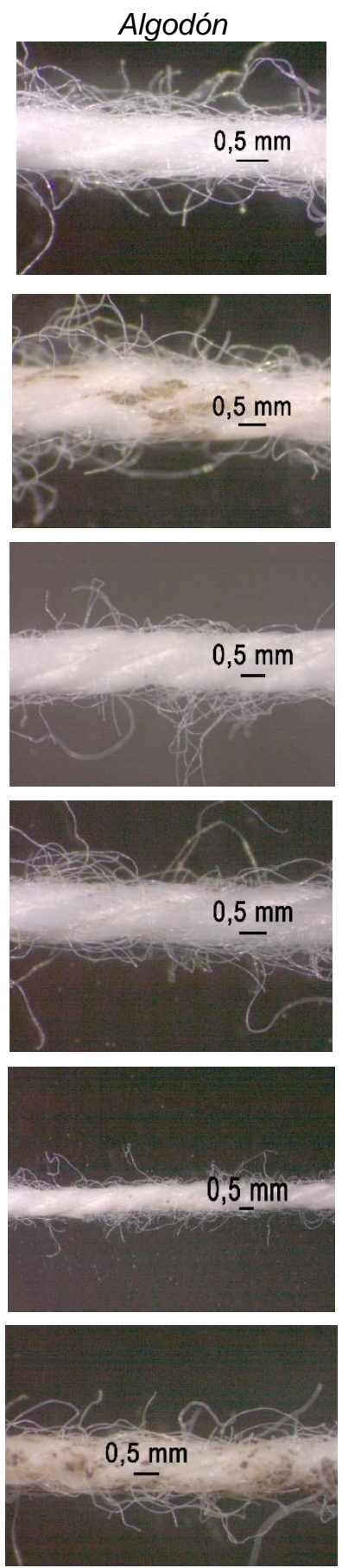

Polipropileno
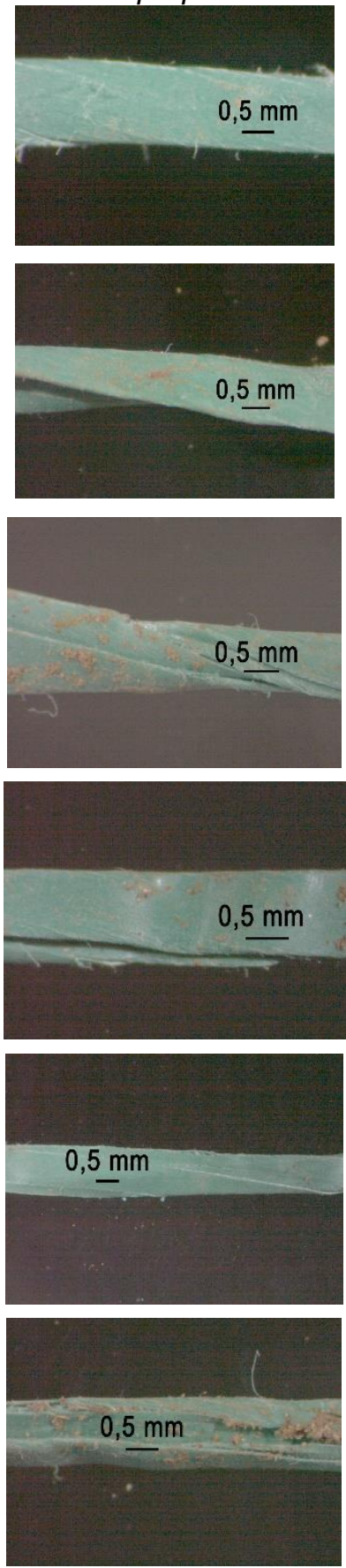

Fig. 5: Observación externa de las fibras algodón y polipropileno

\section{Análisis de textura de las fibras}

En la Fig. 6 se puede observar el análisis de textura correspondiente al módulo de tensión a carga máxima. La fibra de fique perdió rápidamente las propiedades de textura entre los 0 y 16 días pasando de 100 a 26 MPa y después de los 16 días ya no fue posible medir su textura. Las propiedades mecánicas de la fibra de fique cuando está expuesta a condiciones de ambiente reales de suelo se pierden rápidamente. Esto se puede atribuir a las características funcionales y defectos propios de las fibras simples que al final terminan afectando el paquete de fibras y/o organización estructural ( 3 a 12 fibras simples que enrolladas 0 cardadas componen la fibra comercial de fique) (Kicińska-Jakubowska et al. 2012). Adicionalmente, las fibras vegetales naturales son muy heterogéneas y poseen ciertos rasgos anatómicos como aberturas en la pared celular que facilitan el movimiento del agua cuando la planta está viva (Hughes, 2012). Además, posee defectos que facilitan la entrada de humedad y el ataque de microorganismos conocidos como microcompresiones, dislocaciones, ondulaciones y pliegues de la fibra provocados por el proceso de extracción y cardado (Hughes, 2012; Salit, 2014). 
En contraste, las fibras de algodón y polipropileno hasta los 96 días de análisis no pierden sus propiedades mecánicas, incluso se evidencia que la fibra de algodón responde mejor que la fibra de polipropileno. Esto se debe a que, estas fibras son altamente homogéneas en su estructura (Blas-Sevillano et al. 2018; De Silva y Byrne 2017; Jmal et al. 2018). Las propiedades termoplásticas, así como su cristalinidad y grado de polimerización les otorga mayor resistencia al ataque de microorganismos que en su corto tiempo de existencia no son capaces de producir enzimas que desintegren tales estructuras (De Silva y Byrne 2017; Jeon y Kim, 2016; Nair et al. 2017; Jmal et al. 2018). Por otra parte, según Albertsson y Karlsson (1988) durante la biodegradación de fibras altamente resistentes se pueden observar tres etapas de biodegradación correspondientes a (I) una tasa de degradación constante, (II) una disminución parabólica en la tasa de degradación, y (III) un posterior aumento final en la tasa de degradación, no obstante, evidenciar estas tres etapas requieren de mucho tiempo de experimentación ya que todo depende del entorno y de la resistencia de la fibra.

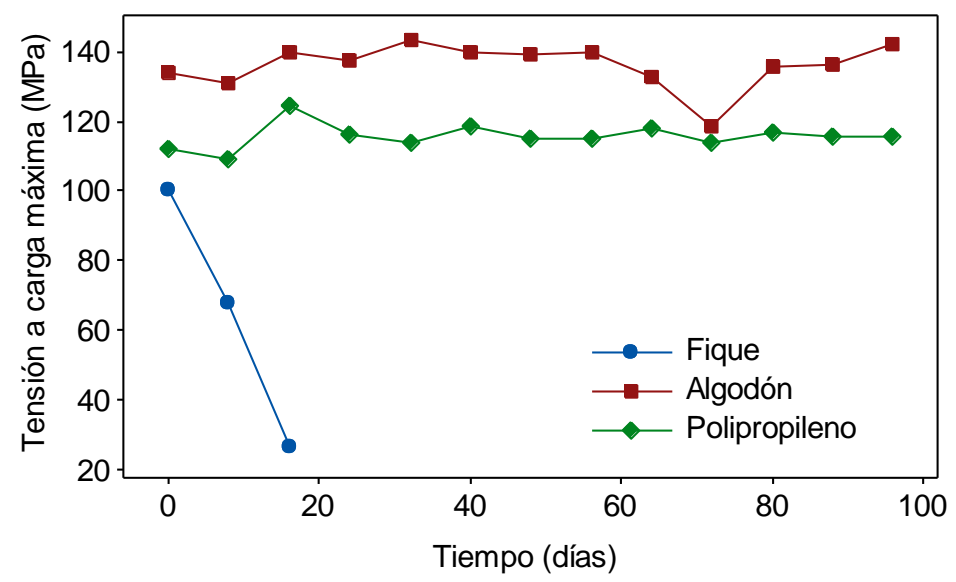

Fig. 6: Análisis de textura durante la biodegradación de fibras a campo abierto

En este sentido, en las fibras de fique, algodón y polipropileno no fue posible observar estas etapas, ya que la fibra de fique enterrada a campo abierto presentó una rápida degradación que afectó sus propiedades mecánicas, debido al prematuro colapso de la estructura. En las fibras de algodón y polipropileno el análisis fue muy corto (96 días) y como sostiene Albertsson y Karlsson (1988) en su análisis de 10 años en fibra de polipropileno apenas alcanzo a apreciar la primera fase (fase de retardo), por esta razón es que en los 96 días de análisis a campo abierto estas dos fibras no presentan cambios significativos, de hecho, el autor sugiere que en este tipo de materiales se debe hacer seguimiento por al menos dos años (Albertsson y Karlsson, 1988; Komilis, 2006).

\section{CONCLUSIONES}

De los resultados mostrados, se pueden obtener las siguientes conclusiones, sobre la biodegradación bajo condiciones contraladas de fibras naturales: 1) Las fibras naturales pueden ser una buena opción de sistemas de tutorado de arveja en términos de biodegradabilidad y ambientales, dado que, una vez terminado su periodo de uso en el tutorado, su degradación e incorporación al suelo seria de solo meses para el fique y de un par de años para el algodón. 2) La fibra de fique presenta mayor facilidad para biodegradarse frente a la fibra de algodón, debido a que, para los microorganismos presentes en el suelo empleado les es más fácil romper la lignina y celulosa del fique que celulosa altamente cristalina del algodón para emplear como fuente de carbono. 3) Dado el tiempo que le toma a la fibra de fique en degradarse resulta en una alternativa para uso agrícola más amigable con el medio ambiente.

\section{AGRADECIMIENTOS}

Agradecimientos al sistema general de regalías - SGR (Colombia) por la financiación del proyecto de "Investigación para el mejoramiento de la tecnología de producción de arveja (Pisum sativum L.) en el departamento de Nariño" proyecto - Cod. BPIN 2013000100278.

\section{REFERENCIAS}

Albertsson, A.C. y S. Karlsson, The three stages in degradation of polymers-polyethylene as a model substance, Journal of Applied Polymer Science, 35(5), 1289-1302 (1988)

Arpitha, G.R. y B. Yogesha, An Overview on Mechanical Property Evaluation of Natural Fiber Reinforced Polymers, Materials Today: Proceedings, 4(2), 2755-2760 (2017) 
ASTM D3822/D3822M-14 Standard Test Method for Tensile Properties of Single Textile Fibers, ASTM International, West Conshohocken, PA (2014)

ASTM D5988 - 12, Standard Test Method for Determining Aerobic Biodegradation of Plastic Materials in Soil1 (2014)

Blas-Sevillano, R.H., T. Veramendi y otros siete autores, Physicochemical characterization of several types of naturally colored cotton fibers from Peru, Carbohydrate Polymers, 197(June), 246-252 (2018)

Chattopadhyay, S.K., S. Singh y otros cinco autores, Biodegradability studies on natural fibers reinforced polypropylene composites, http://doi.wiley.com/10.1002/app.33828, Journal of Applied Polymer Science, 121(4), 2226-2232 (2011)

Coreño-Alonso, J. y M.T. Méndez-Bautista, Relación estructura-propiedades de polímeros, Educación Química, 21(4), 291-299 (2010)

De Silva, R. y N. Byrne, Utilization of cotton waste for regenerated cellulose fibers: Influence of degree of polymerization on mechanical properties, Carbohydrate Polymers, 174, 89-94 (2017)

Emadian, S.M., T.T. Onay y B. Demirel, Biodegradation of bioplastics in natural environments, http://dx.doi.org/10.1016/j.wasman.2016.10.006, Waste Management, Waste Management, 59, 526-536 (2017)

Eriksson KE.L., R.A. Blanchette y P. Ander, Biodegradation of Cellulose, In: Microbial and Enzymatic Degradation of Wood and Wood Components, Springer Series in Wood Science, Springer, Berlin, Heidelberg (1990)

Eubeler, J.P., S. Zok, M. Bernhard y T.P. Knepper, Environmental biodegradation of synthetic polymers I, Test methodologies and procedures, TrAC - Trends in Analytical Chemistry, 28(9), 1057-1072 (2009)

Gómez, E.F. y F.C. Michel, Biodegradability of conventional and bio-based plastics and natural fiber composites during composting, anaerobic digestion and long-term soil incubation, http://dx.doi.org/10.1016/j.polymdegradstab.2013.09.018, Polymer Degradation and Stability, 98(12), 2583-2591 (2013)

Heldman, D.R., 2 - Kinetic Models for Food Systems, In Food Preservation Process Design, Elsevier, 19-48, Academic Press (2011)

Hughes, M., Defects in natural fibers: Their origin, characteristics and implications for natural fiber-reinforced composites, Journal of Materials Science, 47(2), 599-609 (2012)

Jeon, H.J. y M.N. Kim, Isolation of mesophilic bacterium for biodegradation of polypropylene, http://dx.doi.org/10.1016/j.ibiod.2016.08.025, International Biodeterioration and Biodegradation, 115, $244-249$ (2016)

Jmal, H., N. Bahlouli y otros cinco autores, Influence of the grade on the variability of the mechanical properties of polypropylene waste, doi: 10.1016/j.wasman.2018.02.006, Waste Management, 75, 160-173 (2018)

Kicińska-Jakubowska, A., E. Bogacz y M. Zimniewska, Review of Natural Fibers, Part I-Vegetable Fibers, Journal of Natural Fibers, 9(3), 150-167 (2012)

Komilis, D.P., A kinetic analysis of solid waste composting at optimal conditions, Waste Management, 26(1), 82-91 (2006)

Labuza, T.P., Application of chemical kinetics to deterioration of foods, http://pubs.acs.org/doi/abs/10.1021/ed061p348, Journal of Chemical Education, 61(4), 348 (1984)

Leejarkpai, T., U. Suwanmanee, Y. Rudeekit y T. Mungcharoen, Biodegradable kinetics of plastics under controlled composting conditions, http://dx.doi.org/10.1016/j.wasman.2010.12.011, Waste Management, 31(6), 1153-1161 (2011)

Lucas, N., C. Bienaime y otros cuatro autores, Polymer biodegradation: Mechanisms and estimation techniques - A review, Chemosphere, 73(4), 429-442 (2008)

Milošević, M., A. Krkobabić y otros cuatro autores, Biodegradation of cotton and cotton/polyester fabrics impregnated with $\mathrm{Ag} / \mathrm{TiO}_{2}$ nanoparticles in soil, Carbohydrate Polymers, 158, 77-84 (2017)

Nair, N.R., V.C. Sekhar, K.M. Nampoothiri y A. Pandey, Current Developments in Biotechnology and Bioengineering, Chapter 32 - Biodegradation of Biopolymers, 739-755, Elsevier (2016)

Oliveira, T.G., G.L.A. Makishi y otros cuatro autores, Cellulose fiber reinforced biodegradable films based on proteins extracted from castor bean (Ricinus communis L.) cake, Industrial Crops and Products, 67, 355-363 (2015)

Salit, M.S., Tropical Natural Fiber Composites, Properties, Manufacture and Applications, Springer, Singapore (2014)

Scott, G., 12 - Standards for environmentally biodegradable plastics, Biodegradable Polymers for Industrial Applications, 313-335, Woodhead Publishing Limited (2005)

UNCTAD, Biotrade - Designer's Toolkit 1, Materials, Sustainable Biodiversity, Fashion Industry Review, Community Impact. In Fique or Cabuya Furcraea spp. Geneva, United Nations Publication, 12 (2014) 
